\title{
Lagrangian Dynamical Systems with Three Para-complex Structures
}

\author{
Ibrahim Yousif Ibrahim Abad Alrhman \\ Departmentof Mathematics, Faculty of Education, West Kordufan University, Alnhoud City, Sudan \\ Email address: \\ iyibrahimi@gmail.com
}

\section{To cite this article:}

Ibrahim Yousif Ibrahim Abad Alrhman. Lagrangian Dynamical Systems with Three Para-complex Structures. International Journal of Systems Science and Applied Mathematics. Vol. 4, No. 4, 2019, pp. 47-52. doi: 10.11648/j.ijssam.20190404.11

Received: March 21, 2019; Accepted: May 16, 2019; Published: January 17, 2020

\begin{abstract}
This paper aims to present Lagrangian Dynamical systems formalism for mechanical systems using Three ParaComplex Structures, which represent an interesting multidisciplinary field of research. As a result of this study, partial differential equations will be obtained for movement of objects in space and solutions of these equations. In this study, some geometrical, relativistic, mechanical, and physical results related to Three Para- Complex Structures mechanical systems broad applications in mathematical physics, geometrical optics, classical mechanics, analytical mechanics, mechanical systems, thermodynamics, geometric quantization and applied mathematics such as control theory.
\end{abstract}

Keywords: Differential Geometry, Para-complex Structure, Lagrangian Dynamics

\section{Introduction}

The geometric study of dynamical systems is an important chapter of contemporary mathematics due to its applications in Mechanics, Theoretical Physics. If $\mathrm{M}$ is a differentiable manifold that corresponds to the configuration space, a dynamical system can be locally given by a system of ordinary differential equations of the form $\dot{x}^{i}=f^{i}(t ; x)$, which are called equations of evolution. Globally, a dynamical system is givenby a vector field $\mathrm{X}$ on the manifold $M \times R$ whose integral curves, $c(t)$ aregiven by the equations of evolution, $X \circ c(t)=\dot{c}(t)$. The theory of dynamicalsystems dealwith the integration of such systems.

Mehmet and Murat Sari obtained On Para -Euler Lagrange and Para Hamiltonian Equations and constrained para complex Mechanical Equations [1].

Tekkoyun submitted paracomplex analogue of the EulerLagrange equations was obtained in the framework of paraKahlerian manifold and the geometric results on a paracomplex mechanical systems were found [2].

Kasap and Tekkoyun obtained Lagrangian and Hamiltonian formalism for mechanical systems using para/ pseudo-Kahler manifolds, representing an interesting multidisciplinary. field of research. Also, the geometrical, relativistical, mechanical and physical results related to para/ pseudo-Kahler mechanical systems were given, too [3].
Oguzhan and Kasap submitted Mechanical Equations with Two Almost Complex Structures on Symplectic Geometry, using two complex structures, examined mechanical systems on Symplectic geometry [4].

In this paper, we study dynamical systems with Three Almost para Complex Structures. After Introduction in Section 1, we consider Historical Background paper basic. Section 2 deals with the study paracomplex Structures. Section 3 is devoted to study Lagrangian Dynamics.

\section{Preliminaries}

In this preliminary chapter, we recall basic definitions, results and formulas which we shall use in the subsequent chapters of the paper. Most of material included in this chapter occurs in standard literatures namely.

Definition 2.1. [6]

An almost product structure J on a tangent bundle TM of m-real dimensional configurationmanifold $\mathcal{M}$ is a $(1,1)$ tensor field $\mathrm{J}$ on $\mathrm{T} \mathcal{M}$ such that $\mathrm{I}^{2}=\mathrm{I}$. Here, the pair $(\mathrm{T} \mathcal{M}, \mathrm{J})$ is calledan almost product manifold.

Definition 2.2. [8]

An almost Para -complex structure on $\mathcal{M}$ amanifold is a differentiable map I: TM $\rightarrow$ T $\mathcal{M}$ on the tangent bundle $T \mathcal{M}$ of $\mathcal{M}$ such that $I$ preserves each fiber, A manifold with affixed almost para -complex structure is called an almost 
para -complex manifold.

Theorem 2.3. [10]

Suppose that $\left\{x_{1}, x_{2}, x_{3}, x_{4}, x_{5}, x_{6}\right\}$, be a real coordinate system on $(\mathcal{M}, J)$. Then we denote by.

$$
\begin{gathered}
\left\{\frac{\partial}{\partial x_{1}}, \frac{\partial}{\partial x_{2}}, \frac{\partial}{\partial x_{3}}, \frac{\partial}{\partial x_{4}}, \frac{\partial}{\partial x_{5}}, \frac{\partial}{\partial x_{6}}\right\} \\
\left\{d x_{1}, d x_{2}, d x_{3}, d x_{4}, d x_{5}, d x_{6}\right\} \\
J\left(\frac{\partial}{\partial x_{1}}\right)=\frac{\partial}{\partial x_{2}}=j\left(\frac{\partial}{\partial x_{1}}\right), J\left(\frac{\partial}{\partial x_{2}}\right)=\frac{\partial}{\partial x_{1}}=j\left(\frac{\partial}{\partial x_{2}}\right) \\
J\left(\frac{\partial}{\partial x_{3}}\right)=\frac{\partial}{\partial x_{4}}=j\left(\frac{\partial}{\partial x_{3}}\right), J\left(\frac{\partial}{\partial x_{4}}\right)=\frac{\partial}{\partial x_{3}}=j\left(\frac{\partial}{\partial x_{4}}\right) \\
J\left(\frac{\partial}{\partial x_{5}}\right)=\frac{\partial}{\partial x_{6}}=j\left(\frac{\partial}{\partial x_{5}}\right), J\left(\frac{\partial}{\partial x_{6}}\right)=\frac{\partial}{\partial x_{5}}=j\left(\frac{\partial}{\partial x_{6}}\right)
\end{gathered}
$$

\section{Definition 2.4.}

Let $\mathrm{Z}_{1}=\mathrm{x}_{1}+\mathrm{ix}_{2}, \mathrm{Z}_{2}=\mathrm{x}_{3}+\mathrm{ix}_{4}, \mathrm{Z}_{3}=\mathrm{x}_{5}+\mathrm{ix}_{6}, i^{2}=-1$, be Para complex manifold. In local coordinates system on a neighborhood $\mathrm{V}$ of $T \mathcal{M}$. We define the vector fields by.

$$
\begin{aligned}
& I\left(\frac{\partial}{\partial \mathrm{Z}_{1}}\right)=\frac{1}{2}\left(\frac{\partial}{\partial x_{1}}-i \frac{\partial}{\partial x_{2}}\right), I\left(\frac{\partial}{\partial \overline{\mathrm{Z}}_{1}}\right)=\frac{1}{2}\left(\frac{\partial}{\partial x_{1}}+i \frac{\partial}{\partial x_{2}}\right) \\
& I\left(\frac{\partial}{\partial \mathrm{Z}_{2}}\right)=\frac{1}{2}\left(\frac{\partial}{\partial x_{3}}-i \frac{\partial}{\partial x_{4}}\right), I\left(\frac{\partial}{\partial \overline{\mathrm{Z}}_{2}}\right)=\frac{1}{2}\left(\frac{\partial}{\partial x_{3}}+i \frac{\partial}{\partial x_{4}}\right) \\
& I\left(\frac{\partial}{\partial \mathrm{Z}_{3}}\right)=\frac{1}{2}\left(\frac{\partial}{\partial x_{5}}-i \frac{\partial}{\partial x_{6}}\right), I\left(\frac{\partial}{\partial \overline{\mathrm{Z}}_{3}}\right)=\frac{1}{2}\left(\frac{\partial}{\partial x_{5}}+i \frac{\partial}{\partial x_{6}}\right)
\end{aligned}
$$

And the dual convector fields

$$
\begin{aligned}
& I\left(d \mathrm{Z}_{1}\right)=\frac{1}{2}\left(d \mathrm{x}_{1}-i d \mathrm{x}_{2}\right), I\left(d \overline{\mathrm{Z}}_{1}\right)=\frac{1}{2}\left(d \mathrm{x}_{1}-i d \mathrm{x}_{2}\right) \\
& I\left(d \mathrm{Z}_{2}\right)=\frac{1}{2}\left(d \mathrm{x}_{3}-i d \mathrm{x}_{4}\right), I\left(d \overline{\mathrm{Z}}_{2}\right)=\frac{1}{2}\left(d \mathrm{x}_{3}-i d \mathrm{x}_{4}\right) \\
& I\left(d \mathrm{Z}_{3}\right)=\frac{1}{2}\left(d \mathrm{x}_{5}-i d \mathrm{x}_{6}\right), I\left(d \overline{\mathrm{Z}}_{1}\right)=\frac{1}{2}\left(d \mathrm{x}_{5}-i d \mathrm{x}_{6}\right)
\end{aligned}
$$

Theorem 2.5.

Suppose that $\left\{x_{1}, x_{2}, x_{3}, x_{4}, x_{5}, x_{6}\right\}$ be a real coordinate system on $(\mathcal{M}, J)$. Then we denote by.

$$
\begin{array}{r}
\left\{\frac{\partial}{\partial x_{1}}, \frac{\partial}{\partial x_{2}}, \frac{\partial}{\partial x_{3}}, \frac{\partial}{\partial x_{4}}, \frac{\partial}{\partial x_{5}}, \frac{\partial}{\partial x_{6}}\right\} \\
\quad \text { Let } \xi \text { be the vector field by. } \\
\xi=X_{1} \frac{\partial}{\partial x_{1}}+X_{2} \frac{\partial}{\partial x_{2}}+X_{3} \frac{\partial}{\partial x_{3}}+X_{4} \frac{\partial}{\partial x_{4}}+X_{5} \frac{\partial}{\partial x_{5}}+X_{6} \frac{\partial}{\partial x_{6}}
\end{array}
$$

And.

$$
\begin{gathered}
X_{1}=\dot{x}_{1}, X_{2}=\dot{x}_{2}, X_{3}=\dot{x}_{3}, X_{4}=\dot{x}_{4}, X_{5}=\dot{x}_{5}, X_{6}=\dot{x}_{6} \\
J(\xi)=J\left(X_{1} \frac{\partial}{\partial x_{1}}+X_{2} \frac{\partial}{\partial x_{2}}+X_{3} \frac{\partial}{\partial x_{3}}+X_{4} \frac{\partial}{\partial x_{4}}+X_{5} \frac{\partial}{\partial x_{5}}+X_{6} \frac{\partial}{\partial x_{6}}\right) \\
J(\xi)=X_{1} J\left(\frac{\partial}{\partial x_{1}}\right)+X_{2} J\left(\frac{\partial}{\partial x_{2}}\right)+X_{3} J\left(\frac{\partial}{\partial x_{3}}\right)+X_{4} J\left(\frac{\partial}{\partial x_{4}}\right)+X_{5} J\left(\frac{\partial}{\partial x_{5}}\right)+X_{6} J\left(\frac{\partial}{\partial x_{6}}\right)
\end{gathered}
$$

If $\mathrm{I}$ is defined as a Para complex manifold $\mathcal{M}$ then $I^{2}=\mathrm{I}$ 。

$$
\begin{aligned}
& I^{2}\left(\frac{\partial}{\partial x_{1}}\right)=\mathrm{I}\left(\mathrm{I}\left(\frac{\partial}{\partial x_{1}}\right)\right)=\mathrm{I}\left(\frac{\partial}{\partial x_{2}}\right)=\frac{\partial}{\partial x_{1}} \\
& I^{2}\left(\frac{\partial}{\partial x_{2}}\right)=\mathrm{I}\left(\mathrm{I}\left(\frac{\partial}{\partial x_{2}}\right)\right)=\mathrm{I}\left(\frac{\partial}{\partial x_{1}}\right)=\frac{\partial}{\partial x_{2}} \\
& I^{2}\left(\frac{\partial}{\partial x_{3}}\right)=\mathrm{I}\left(\mathrm{I}\left(\frac{\partial}{\partial x_{3}}\right)\right)=\mathrm{I}\left(\frac{\partial}{\partial x_{4}}\right)=\frac{\partial}{\partial x_{3}} \\
& I^{2}\left(\frac{\partial}{\partial x_{4}}\right)=\mathrm{I}\left(\mathrm{I}\left(\frac{\partial}{\partial x_{4}}\right)\right)=\mathrm{I}\left(\frac{\partial}{\partial x_{3}}\right)=\frac{\partial}{\partial x_{4}} \\
& I^{2}\left(\frac{\partial}{\partial x_{5}}\right)=\mathrm{I}\left(\mathrm{I}\left(\frac{\partial}{\partial x_{5}}\right)\right)=\mathrm{I}\left(\frac{\partial}{\partial x_{6}}\right)=\frac{\partial}{\partial x_{5}} \\
& I^{2}\left(\frac{\partial}{\partial x_{6}}\right)=\mathrm{I}\left(\mathrm{I}\left(\frac{\partial}{\partial x_{6}}\right)\right)=\mathrm{I}\left(\frac{\partial}{\partial x_{5}}\right)=\frac{\partial}{\partial x_{6}}
\end{aligned}
$$

\section{Lagrangian Dynamical Systems}

In this section we introduce the concept of Lagrangian

Definition 3.1. [5]

A Lagrangian function vector field $\mathrm{X}$ on $\mathcal{M}$ is a smooth function $\mathrm{L}: \mathrm{T} \mathcal{M} \rightarrow \mathrm{R}$ such that.

$$
\mathrm{i}_{\mathrm{X}} \phi_{\mathrm{L}}=\mathrm{dE}_{\mathrm{L}}
$$




$$
U=J(\xi)=-X_{1} j \frac{\partial}{\partial x_{1}}+X_{2} j \frac{\partial}{\partial x_{2}}-X_{3} j \frac{\partial}{\partial x_{3}}+X_{4} j \frac{\partial}{\partial x_{4}}-X_{5} j \frac{\partial}{\partial x_{5}}+X_{6} j \frac{\partial}{\partial x_{6}}
$$

Let that Liouville Vector field on complex manifold $(\mathcal{M}, U)$.

Definition 3.2. [11]

Kinetic energy given $T: T \mathcal{M} \rightarrow \mathcal{M}$.

$$
T=\frac{1}{2} m_{i}\left(\dot{x}_{1}^{2}+\dot{x}_{2}^{2}+\dot{x}_{3}^{2}+\dot{x}_{4}^{2}+\dot{x}_{5}^{2}+\dot{x}_{6}^{2}\right)
$$

Potential energy $P: T \mathcal{M} \rightarrow \mathcal{M}$.

$$
P=m_{i} g h
$$

Definition 3.3. [14]

The Lagrangian function (energy function)

$$
\begin{gathered}
L=T-P \\
E_{L}^{J}=U_{G_{1}}(L)-L
\end{gathered}
$$

Definition 3.4. [2]

Is vertical derivation (differentiation) $d_{J}$ is defined

$$
d_{G_{J}}=\left[i_{G_{J}}, d\right]=i_{G_{J}} d-d i_{J}
$$

$\phi_{\mathrm{L}}=d d_{1} L$ such that.

$$
d_{J}=\frac{\partial}{\partial x_{2}} d x_{1}-\frac{\partial}{\partial x_{1}} d x_{2}+\frac{\partial}{\partial x_{4}} d x_{3}-\frac{\partial}{\partial x_{3}} d x_{4}+\frac{\partial}{\partial x_{6}} d x_{5}-\frac{\partial}{\partial x_{5}} d x_{6}
$$

Defined by operatord $d_{J}: A(\mathcal{M}) \rightarrow \Lambda^{1} \mathcal{M}$

$$
\begin{array}{r}
d_{J} L=\left(\frac{\partial}{\partial x_{2}} d x_{1}-\frac{\partial}{\partial x_{1}} d x_{2}+\frac{\partial}{\partial x_{4}} d x_{3}-\frac{\partial}{\partial x_{3}} d x_{4}+\frac{\partial}{\partial x_{6}} d x_{5}-\frac{\partial}{\partial x_{5}} d x_{6}\right) L \\
d_{J} L=-\boldsymbol{j} \frac{\partial L}{\partial x_{2}} d x_{1}+\boldsymbol{j} \frac{\partial L}{\partial x_{1}} d x_{2}-\boldsymbol{j} \frac{\partial L}{\partial x_{4}} d x_{3}+\boldsymbol{j} \frac{\partial L}{\partial x_{3}} d x_{4}-\boldsymbol{j} \frac{\partial L}{\partial x_{6}} d x_{5}+\boldsymbol{j} \frac{\partial L}{\partial x_{5}} d x_{6}
\end{array}
$$

That.

$$
\begin{aligned}
& \phi_{\mathrm{L}}=-d\left(d_{G_{1}}\right)=-d\left(-\boldsymbol{j} \frac{\partial L}{\partial x_{2}} d x_{1}+\boldsymbol{j} \frac{\partial L}{\partial x_{1}} d x_{2}-\boldsymbol{j} \frac{\partial L}{\partial x_{4}} d x_{3}+\boldsymbol{j} \frac{\partial L}{\partial x_{3}} d x_{4}-\boldsymbol{j} \frac{\partial L}{\partial x_{6}} d x_{5}+\boldsymbol{j} \frac{\partial L}{\partial x_{5}} d x_{6}\right) \\
& \phi_{\mathrm{L}}=\boldsymbol{j} \frac{\partial^{2} L}{\partial x_{1} \partial x_{2}} d x_{1} \wedge d x_{1}-\boldsymbol{j} \frac{\partial^{2} L}{\partial x_{1} \partial x_{1}} d x_{1} \wedge d x_{2}+\boldsymbol{j} \frac{\partial^{2} L}{\partial x_{1} \partial x_{4}} d x_{1} \wedge d x_{3}-\boldsymbol{j} \frac{\partial^{2} L}{\partial x_{1} \partial x_{3}} d x_{1} \wedge d x_{4}+\boldsymbol{j} \frac{\partial^{2} L}{\partial x_{1} \partial x_{6}} d x_{1} \wedge d x_{5} \\
& \quad-\boldsymbol{j} \frac{\partial^{2} L}{\partial x_{1} \partial x_{5}} d x_{1} \wedge d x_{6}+\boldsymbol{j} \frac{\partial^{2} L}{\partial x_{2} \partial x_{2}} d x_{2} \wedge d x_{1}-\boldsymbol{j} \frac{\partial^{2} L}{\partial x_{2} \partial x_{1}} d x_{2} \wedge d x_{2}+\boldsymbol{j} \frac{\partial^{2} L}{\partial x_{2} \partial x_{4}} d x_{2} \wedge d x_{3}-\boldsymbol{j} \frac{\partial^{2} L}{\partial x_{2} \partial x_{3}} d x_{2} \\
& \wedge d x_{4}+\boldsymbol{j} \frac{\partial^{2} L}{\partial x_{2} \partial x_{6}} d x_{2} \wedge d x_{5}-\boldsymbol{j} \frac{\partial^{2} L}{\partial x_{2} \partial x_{5}} d x_{2} \wedge d x_{6}+\boldsymbol{j} \frac{\partial^{2} L}{\partial x_{3} \partial x_{2}} d x_{3} \wedge d x_{1}-\boldsymbol{j} \frac{\partial^{2} L}{\partial x_{3} \partial x_{1}} d x_{3} \wedge d x_{2} \\
&+\boldsymbol{j} \frac{\partial^{2} L}{\partial x_{3} \partial x_{4}} d x_{3} \wedge d x_{3}-\boldsymbol{j} \frac{\partial^{2} L}{\partial x_{3} \partial x_{3}} d x_{3} \wedge d x_{4}+\boldsymbol{j} \frac{\partial^{2} L}{\partial x_{3} \partial x_{6}} d x_{3} \wedge d x_{5}-\boldsymbol{j} \frac{\partial^{2} L}{\partial x_{3} \partial x_{5}} d x_{3} \wedge d x_{6}+\boldsymbol{j} \boldsymbol{v} \frac{\partial^{2} L}{\partial x_{4} \partial x_{2}} d x_{4} \\
& \wedge d x_{1}-\boldsymbol{j} \frac{\partial^{2} L}{\partial x_{4} \partial x_{1}} d x_{4} \wedge d x_{2}+\boldsymbol{j} \frac{\partial^{2} L}{\partial x_{4} \partial x_{4}} d x_{4} \wedge d x_{3}-\boldsymbol{j} \frac{\partial^{2} L}{\partial x_{4} \partial x_{3}} d x_{4} \wedge d x_{4}+\boldsymbol{j} \frac{\partial^{2} L}{\partial x_{4} \partial x_{6}} d x_{4} \wedge d x_{5} \\
& \quad-\boldsymbol{j} \frac{\partial^{2} L}{\partial x_{4} \partial x_{5}} d x_{4} \wedge d x_{6}+\boldsymbol{j} \frac{\partial^{2} L}{\partial x_{5} \partial x_{2}} d x_{5} \wedge d x_{1}-\boldsymbol{j} \frac{\partial^{2} L}{\partial x_{5} \partial x_{1}} d x_{5} \wedge d x_{2}+\boldsymbol{j} \frac{\partial^{2} L}{\partial x_{5} \partial x_{4}} d x_{5} \wedge d x_{3}-\boldsymbol{j} \frac{\partial^{2} L}{\partial x_{5} \partial x_{3}} d x_{5} \\
& \wedge d x_{4}+\boldsymbol{j} \frac{\partial^{2} L}{\partial x_{5} \partial x_{6}} d x_{5} \wedge d x_{5}-\boldsymbol{j} \frac{\partial^{2} L}{\partial x_{5} \partial x_{5}} d x_{5} \wedge d x_{6}+\boldsymbol{j} \frac{\partial^{2} L}{\partial x_{6} \partial x_{2}} d x_{6} \wedge d x_{1}-\boldsymbol{j} \frac{\partial^{2} L}{\partial x_{6} \partial x_{1}} d x_{6} \wedge d x_{2} \\
&+\boldsymbol{j} \frac{\partial^{2} L}{\partial x_{6} \partial x_{4}} d x_{6} \wedge d x_{3}-\boldsymbol{j} \frac{\partial^{2} L}{\partial x_{6} \partial x_{3}} d x_{6} \wedge d x_{4}+\boldsymbol{j} \frac{\partial^{2} L}{\partial x_{6} \partial x_{6}} d x_{6} \wedge d x_{5}-\boldsymbol{j} \frac{\partial^{2} L}{\partial x_{6} \partial x_{5}} d x_{6} \wedge d x_{6}
\end{aligned}
$$

Calculate $\phi_{\mathrm{L}}(\xi)$. 
$\mathrm{i}_{\mathrm{X}} \phi_{\mathrm{L}}=\phi_{\mathrm{L}}(\xi)=\left(j \frac{\partial^{2} L}{\partial x_{1} \partial x_{2}} d x_{1} \wedge d x_{1}-j \frac{\partial^{2} L}{\partial x_{1} \partial x_{1}} d x_{1} \wedge d x_{2}+j \frac{\partial^{2} L}{\partial x_{1} \partial x_{4}} d x_{1} \wedge d x_{3}-j \frac{\partial^{2} L}{\partial x_{1} \partial x_{3}} d x_{1} \wedge d x_{4}+j \frac{\partial^{2} L}{\partial x_{1} \partial x_{6}} d x_{1} \wedge d x_{5}-\right.$ $j \frac{\partial^{2} L}{\partial x_{1} \partial x_{5}} d x_{1} \wedge d x_{6}+j \frac{\partial^{2} L}{\partial x_{2} \partial x_{2}} d x_{2} \wedge d x_{1}-j \frac{\partial^{2} L}{\partial x_{2} \partial x_{1}} d x_{2} \wedge d x_{2}+j \frac{\partial^{2} L}{\partial x_{2} \partial x_{4}} d x_{2} \wedge d x_{3}-j \frac{\partial^{2} L}{\partial x_{2} \partial x_{3}} d x_{2} \wedge d x_{4}+j \frac{\partial^{2} L}{\partial x_{2} \partial x_{6}} d x_{2} \wedge$ $d x_{5}-j \frac{\partial^{2} L}{\partial x_{2} \partial x_{5}} d x_{2} \wedge d x_{6}+j \frac{\partial^{2} L}{\partial x_{3} \partial x_{2}} d x_{3} \wedge d x_{1}-j \frac{\partial^{2} L}{\partial x_{3} \partial x_{1}} d x_{3} \wedge d x_{2}+j \frac{\partial^{2} L}{\partial x_{3} \partial x_{4}} d x_{3} \wedge d x_{3}-j \frac{\partial^{2} L}{\partial x_{3} \partial x_{3}} d x_{3} \wedge d x_{4}+$ $j \frac{\partial^{2} L}{\partial x_{3} \partial x_{6}} d x_{3} \wedge d x_{5}-j \frac{\partial^{2} L}{\partial x_{3} \partial x_{5}} d x_{3} \wedge d x_{6}+j \frac{\partial^{2} L}{\partial x_{4} \partial x_{2}} d x_{4} \wedge d x_{1}-j \frac{\partial^{2} L}{\partial x_{4} \partial x_{1}} d x_{4} \wedge d x_{2}+j \frac{\partial^{2} L}{\partial x_{4} \partial x_{4}} d x_{4} \wedge d x_{3}-j \frac{\partial^{2} L}{\partial x_{4} \partial x_{3}} d x_{4} \wedge$ $d x_{4}+j \frac{\partial^{2} L}{\partial x_{4} \partial x_{6}} d x_{4} \wedge d x_{5}-j \frac{\partial^{2} L}{\partial x_{4} \partial x_{5}} d x_{4} \wedge d x_{6}+j \frac{\partial^{2} L}{\partial x_{5} \partial x_{2}} d x_{5} \wedge d x_{1}-j \frac{\partial^{2} L}{\partial x_{5} \partial x_{1}} d x_{5} \wedge d x_{2}+j \frac{\partial^{2} L}{\partial x_{5} \partial x_{4}} d x_{5} \wedge d x_{3}+\frac{\partial^{2} L}{\partial x_{5} \partial x_{3}} d x_{5} \wedge$ $d x_{4}-\frac{\partial^{2} L}{\partial x_{5} \partial x_{6}} d x_{5} \wedge d x_{5}+\frac{\partial^{2} L}{\partial x_{5} \partial x_{5}} d x_{5} \wedge d x_{6}-\frac{\partial^{2} L}{\partial x_{6} \partial x_{2}} d x_{6} \wedge d x_{1}+\frac{\partial^{2} L}{\partial x_{6} \partial x_{1}} d x_{6} \wedge d x_{2}+j \frac{\partial^{2} L}{\partial x_{6} \partial x_{4}} d x_{6} \wedge d x_{3}-j \frac{\partial^{2} L}{\partial x_{6} \partial x_{3}} d x_{6} \wedge$ $\left.d x_{4}+j \frac{\partial^{2} L}{\partial x_{6} \partial x_{6}} d x_{6} \wedge d x_{5}-j \frac{\partial^{2} L}{\partial x_{6} \partial x_{5}} d x_{6} \wedge d x_{6}\right)\left(X^{1} \frac{\partial}{\partial x_{1}}+X^{2} \frac{\partial}{\partial x_{2}}+X^{3} \frac{\partial}{\partial x_{3}}+X^{4} \frac{\partial}{\partial x_{4}}+X^{5} \frac{\partial}{\partial x_{5}}+X^{6} \frac{\partial}{\partial x_{6}}\right)$

From the energy equation we get.

$$
E_{L}=V(L)-L=X^{1} \frac{\partial L}{\partial x_{2}}-X^{2} \frac{\partial L}{\partial x_{1}}+X^{3} \frac{\partial L}{\partial x_{4}}-X^{4} \frac{\partial L}{\partial x_{3}}+X^{5} \frac{\partial L}{\partial x_{6}}-X^{6} \frac{\partial L}{\partial x_{5}}-L
$$

In the equation of the energy equation we obtain.

$$
\begin{aligned}
& d E_{L}=\left(-j \frac{\partial}{\partial x_{2}} d x_{1}+j \frac{\partial}{\partial x_{1}} d x_{2}-j \frac{\partial}{\partial x_{4}} d x_{3}+j \frac{\partial}{\partial x_{3}} d x_{4}-j \frac{\partial}{\partial x_{6}} d x_{5}+j \frac{\partial}{\partial x_{5}} d x_{6}\right)\left(X^{1} \frac{\partial L}{\partial x_{2}}-X^{2} \frac{\partial L}{\partial x_{1}}+X^{3} \frac{\partial L}{\partial x_{4}}-X^{4} \frac{\partial L}{\partial x_{3}}\right. \\
& \left.+X^{5} \frac{\partial L}{\partial x_{6}}-X^{6} \frac{\partial L}{\partial x_{5}}-L\right) \\
& d E_{L}=-j X^{1} \frac{\partial^{2} L}{\partial x_{1} \partial x_{2}} d x_{1}-j X^{1} \frac{\partial^{2} L}{\partial x_{2} \partial x_{2}} d x_{2}-j X^{1} \frac{\partial^{2} L}{\partial x_{3} \partial x_{2}} d x_{3}-j X^{1} \frac{\partial^{2} L}{\partial x_{4} \partial x_{2}} d x_{4}-j X^{1} \frac{\partial^{2} L}{\partial x_{5} \partial x_{2}} d x_{5}-X^{1} \frac{\partial^{2} L}{\partial x_{6} \partial x_{2}} d x_{6} \\
& +j X^{2} \frac{\partial^{2} L}{\partial x_{1} \partial x_{1}} d x_{1}+j X^{2} \frac{\partial^{2} L}{\partial x_{2} \partial x_{1}} d x_{2}+j X^{2} \frac{\partial^{2} L}{\partial x_{3} \partial x_{1}} d x_{3}+j X^{2} \frac{\partial^{2} L}{\partial x_{4} \partial x_{1}} d x_{4}+j X^{2} \frac{\partial^{2} L}{\partial x_{5} \partial x_{1}} d x_{5}+j X^{2} \frac{\partial^{2} L}{\partial x_{6} \partial x_{1}} d x_{6} \\
& -j X^{3} \frac{\partial^{2} L}{\partial x_{1} \partial x_{4}} d x_{1}-j X^{3} \frac{\partial^{2} L}{\partial x_{2} \partial x_{4}} d x_{2}-j X^{3} \frac{\partial^{2} L}{\partial x_{3} \partial x_{4}} d x_{3}-j X^{3} \frac{\partial^{2} L}{\partial x_{4} \partial x_{4}} d x_{4}-j X^{3} \frac{\partial^{2} L}{\partial x_{5} \partial x_{4}} d x_{5}-j X^{3} \frac{\partial^{2} L}{\partial x_{6} \partial x_{4}} d x_{6} \\
& +j X^{4} \frac{\partial^{2} L}{\partial x_{1} \partial x_{3}} d x_{1}+j X^{4} \frac{\partial^{2} L}{\partial x_{2} \partial x_{3}} d x_{2}+j X^{4} \frac{\partial^{2} L}{\partial x_{3} \partial x_{3}} d x_{3}+j X^{4} \frac{\partial^{2} L}{\partial x_{4} \partial x_{3}} d x_{4}+j X^{4} \frac{\partial^{2} L}{\partial x_{5} \partial x_{3}} d x_{5}+j X^{4} \frac{\partial^{2} L}{\partial x_{6} \partial x_{3}} d x_{6} \\
& -j X^{5} \frac{\partial^{2} L}{\partial x_{1} \partial x_{6}} d x_{1}-j X^{5} \frac{\partial^{2} L}{\partial x_{2} \partial x_{6}} d x_{2}-j X^{5} \frac{\partial^{2} L}{\partial x_{3} \partial x_{6}} d x_{3}-j X^{5} \frac{\partial^{2} L}{\partial x_{4} \partial x_{6}} d x_{4}-j X^{5} \frac{\partial^{2} L}{\partial x_{5} \partial x_{6}} d x_{5}-j X^{5} \frac{\partial^{2} L}{\partial x_{6} \partial x_{6}} d x_{6} \\
& +j X^{6} \frac{\partial^{2} L}{\partial x_{1} \partial x_{5}} d x_{1}+j X^{6} \frac{\partial^{2} L}{\partial x_{2} \partial x_{5}} d x_{2}+j X^{6} \frac{\partial^{2} L}{\partial x_{3} \partial x_{5}} d x_{3}+j X^{6} \frac{\partial^{2} L}{\partial x_{4} \partial x_{5}} d x_{4}+j X^{6} \frac{\partial^{2} L}{\partial x_{5} \partial x_{5}} d x_{5}+j X^{6} \frac{\partial^{2} L}{\partial x_{6} \partial x_{5}} d x_{6} \\
& -\frac{\partial L}{\partial x_{1}} d x_{1}-\frac{\partial L}{\partial x_{2}} d x_{2}-\frac{\partial L}{\partial x_{3}} d x_{3}-\frac{\partial L}{\partial x_{4}} d x_{4}-\frac{\partial L}{\partial x_{5}} d x_{5}-\frac{\partial L}{\partial x_{6}} d x_{6}
\end{aligned}
$$

Equation of Equation (8) with Equation (9) we obtain.

$$
\begin{gathered}
\mathrm{i}_{X} \phi_{\mathrm{L}}=d E_{L} \\
j\left(X^{1} \frac{\partial}{\partial x_{1}}+X^{1} \frac{\partial}{\partial x_{2}}+X^{1} \frac{\partial}{\partial x_{3}}+X^{1} \frac{\partial}{\partial x_{4}}+X^{1} \frac{\partial}{\partial x_{5}} d x_{5}+X^{1} \frac{\partial}{\partial x_{6}}\right)\left(\frac{\partial L}{\partial x_{2}}\right) d x_{1}+\frac{\partial L}{\partial x_{1}} d x_{1} \\
-j\left(X^{1} \frac{\partial}{\partial x_{1}}+X^{1} \frac{\partial}{\partial x_{2}}+X^{1} \frac{\partial}{\partial x_{3}}+X^{1} \frac{\partial}{\partial x_{4}}+X^{1} \frac{\partial}{\partial x_{5}} d x_{5}+X^{1} \frac{\partial}{\partial x_{6}}\right)\left(\frac{\partial L}{\partial x_{1}}\right) d x_{2}+\frac{\partial L}{\partial x_{2}} d x_{2} \\
+j\left(X^{1} \frac{\partial}{\partial x_{1}}+X^{1} \frac{\partial}{\partial x_{2}}+X^{1} \frac{\partial}{\partial x_{3}}+X^{1} \frac{\partial}{\partial x_{4}}+X^{1} \frac{\partial}{\partial x_{5}} d x_{5}+X^{1} \frac{\partial}{\partial x_{6}}\right)\left(\frac{\partial L}{\partial x_{3}}\right) d x_{3}+\frac{\partial L}{\partial x_{3}} d x_{3} \\
-j\left(X^{1} \frac{\partial}{\partial x_{1}}+X^{1} \frac{\partial}{\partial x_{2}}+X^{1} \frac{\partial}{\partial x_{3}}+X^{1} \frac{\partial}{\partial x_{4}}+X^{1} \frac{\partial}{\partial x_{5}} d x_{5}+X^{1} \frac{\partial}{\partial x_{6}}\right)\left(\frac{\partial L}{\partial x_{4}}\right) d x_{4}+\frac{\partial L}{\partial x_{4}} d x_{4}
\end{gathered}
$$




$$
\begin{gathered}
j\left(X^{1} \frac{\partial}{\partial x_{1}}+X^{1} \frac{\partial}{\partial x_{2}}+X^{1} \frac{\partial}{\partial x_{3}}+X^{1} \frac{\partial}{\partial x_{4}}+X^{1} \frac{\partial}{\partial x_{5}} d x_{5}+X^{1} \frac{\partial}{\partial x_{6}}\right)\left(\frac{\partial L}{\partial x_{5}}\right) d x_{5}+\frac{\partial L}{\partial x_{5}} d x_{5} \\
-j\left(X^{1} \frac{\partial}{\partial x_{1}}+X^{1} \frac{\partial}{\partial x_{2}}+X^{1} \frac{\partial}{\partial x_{3}}+X^{1} \frac{\partial}{\partial x_{4}}+X^{1} \frac{\partial}{\partial x_{5}} d x_{5}+X^{1} \frac{\partial}{\partial x_{6}}\right)\left(\frac{\partial L}{\partial x_{6}}\right) d x_{6}+\frac{\partial L}{\partial x_{6}} d x_{6}=0
\end{gathered}
$$

Be an integral curve. in local coordinates it is obtained that.

Suppose that a curve.

$$
\alpha: \mathrm{I} \subset \mathrm{R} \rightarrow \mathrm{T}^{*} \mathcal{M}=R^{2 n}
$$

is an integral curve of the Lagrangian vector field $\mathrm{X}_{\mathrm{H}}$, i. e.,

$$
X_{L}(\alpha(t))=\frac{d \alpha(t)}{d t}, t \in I
$$

In the local coordinates, if it is considered to be

$$
\alpha(t)=\left(x_{1}(t), x_{2}(t), x_{2}(t), x_{4}(t), x_{5}(t), x_{6}(t)\right)
$$

we obtain

$$
\frac{\mathrm{d} \alpha(\mathrm{t})}{\mathrm{dt}}=\frac{\mathrm{dx}_{1}}{\mathrm{dt}} \frac{\partial}{\partial \mathrm{x}_{1}}+\frac{\mathrm{dx}_{2}}{\mathrm{dt}} \frac{\partial}{\partial \mathrm{x}_{2}}+\frac{\mathrm{dx}_{3}}{\mathrm{dt}} \frac{\partial}{\partial \mathrm{x}_{3}}+\frac{\mathrm{dx}_{4}}{\mathrm{dt}} \frac{\partial}{\partial \mathrm{x}_{4}}+\frac{\mathrm{dx}_{5}}{\mathrm{dt}} \frac{\partial}{\partial \mathrm{x}_{5}}+\frac{\mathrm{dx}_{6}}{\mathrm{dt}} \frac{\partial}{\partial \mathrm{x}_{6}} X^{1} \frac{\partial}{\partial x_{1}}+X^{1} \frac{\partial}{\partial x_{2}}+X^{1} \frac{\partial}{\partial x_{3}}+X^{1} \frac{\partial}{\partial x_{4}}+X^{1} \frac{\partial}{\partial x_{5}} d x_{5}+X^{1} \frac{\partial}{\partial x_{6}}=\frac{\partial}{\partial \mathrm{t}}
$$

Taking the equation $(10)=$ the equation (11).

$$
\begin{gathered}
j \frac{\partial}{\partial \mathrm{t}}\left(\frac{\partial L}{\partial x_{2}}\right) d x_{1}+\frac{\partial L}{\partial x_{1}} d x_{1}=0 \rightarrow j \frac{\partial}{\partial \mathrm{t}}\left(\frac{\partial L}{\partial x_{2}}\right)+\frac{\partial L}{\partial x_{1}}=0 \\
-j \frac{\partial}{\partial \mathrm{t}}\left(\frac{\partial L}{\partial x_{1}}\right) d x_{2}+\frac{\partial L}{\partial x_{2}} d x_{2}=0 \rightarrow j \frac{\partial}{\partial \mathrm{t}}\left(\frac{\partial L}{\partial x_{1}}\right)-\frac{\partial L}{\partial x_{2}}=0 \\
j \frac{\partial}{\partial \mathrm{t}}\left(\frac{\partial L}{\partial x_{3}}\right) d x_{3}+\frac{\partial L}{\partial x_{3}} d x_{3}=0 \rightarrow j \frac{\partial}{\partial \mathrm{t}}\left(\frac{\partial L}{\partial x_{3}}\right)+\frac{\partial L}{\partial x_{3}}=0 \\
-j \frac{\partial}{\partial \mathrm{t}}\left(\frac{\partial L}{\partial x_{4}}\right) d x_{4}+\frac{\partial L}{\partial x_{4}} d x_{4}=0 \rightarrow j \frac{\partial}{\partial \mathrm{t}}\left(\frac{\partial L}{\partial x_{4}}\right)-\frac{\partial L}{\partial x_{4}}=0 \\
j \frac{\partial}{\partial \mathrm{t}}\left(\frac{\partial L}{\partial x_{5}}\right) d x_{5}+\frac{\partial L}{\partial x_{5}} d x_{5}=0 \rightarrow j \frac{\partial}{\partial \mathrm{t}}\left(\frac{\partial L}{\partial x_{5}}\right)+\frac{\partial L}{\partial x_{5}}=0 \\
-j \frac{\partial}{\partial \mathrm{t}}\left(\frac{\partial L}{\partial x_{6}}\right) d x_{6}+\frac{\partial L}{\partial x_{6}} d x_{6}=0 \rightarrow j \frac{\partial}{\partial \mathrm{t}}\left(\frac{\partial L}{\partial x_{6}}\right)-\frac{\partial L}{\partial x_{6}}=0
\end{gathered}
$$

And.

$$
\begin{aligned}
& j \frac{\partial}{\partial \mathrm{t}}\left(\frac{\partial L}{\partial x_{2}}\right)+\frac{\partial L}{\partial x_{1}}=0, j \frac{\partial}{\partial \mathrm{t}}\left(\frac{\partial L}{\partial x_{1}}\right)-\frac{\partial L}{\partial x_{2}}=0, j \frac{\partial}{\partial \mathrm{t}}\left(\frac{\partial L}{\partial x_{3}}\right)+\frac{\partial L}{\partial x_{3}}=0 \\
& j \frac{\partial}{\partial \mathrm{t}}\left(\frac{\partial L}{\partial x_{4}}\right)-\frac{\partial L}{\partial x_{4}}=0, j \frac{\partial}{\partial \mathrm{t}}\left(\frac{\partial L}{\partial x_{5}}\right)+\frac{\partial L}{\partial x_{5}}=0, j \frac{\partial}{\partial \mathrm{t}}\left(\frac{\partial L}{\partial x_{6}}\right)-\frac{\partial L}{\partial x_{6}}=0
\end{aligned}
$$

Hence the triple $\left(\mathcal{M}, \phi_{\mathrm{L}}, \xi\right)$ is called apara complex Lagrangian mechanical system which are deduced by means of an almost real structure $\mathrm{j}$ and using of basis $\left\{\frac{\partial}{\partial \mathrm{x}_{\mathrm{i}}}: \mathrm{i}=\right.$ $1,2,3,4,5,6\}$ on the distributions $\mathcal{M}$.

\section{Conclusions}

From the study, we obtain that Lagrangian formalisms in generalized Classical Mechanics and field theory can be intrinsically characterized on $\left(\mathrm{T} \mathcal{M}, \phi_{\mathrm{L}}, \xi\right)$ being a model of
Three Para-Complex Structures. So, the paths of semispray $\xi$ on $\mathrm{J}^{(2,0)} \mathcal{M}$ are the solutions of the Euler-Lagrange equations given by (12) on the mechanical system $\left(\mathrm{T} \mathcal{M}, \phi_{\mathrm{L}}, \xi\right)$.

\section{References}

[1] Mehmet Tekkoyun, On Para -Euler Lagrange and Para Hamiltonian Equations, physics letters A0 340 (2005) pp7-11.

[2] Mehmet Mekkoyun and Murat Sari- Bi-Para Mechanical Equations on Lagrangian Distributions -arXivo: 0902. v1 [math. Ph] 8Jan 2009. 
[3] Mehmet Mekkoyun and Murat Sari Constrained para complex Mechanical Equations -arXivo: 0902-41210. v1 [math. DS] 24Feb 2009.

[4] Oguzhan Celik and Zeki Kasap, Mechanical Equations with Two Almost Complex Structures on Symplectic Geometry, April 28, 2016.

[5] Zeki Kasap and Mehmet Mekkoyun, Mechanical Equations on Bi-Para Conformal arXivo1209, 3101. V2 [math. GM] 22Sep 2010.

[6] http//en.Wikipedia.org/wiki/almost complex structure.

[7] Cristian Ida, Alexandru Ionescu and Adelina Manea, A note on para-holomorphic Riemannian Einstein manifolds, arXiv: 1507. 01114v2 [math. DG] 19 May 2016.

[8] Z. Kasap and M. Tekkoyun, Mechanical Systems on Almost Para/ Pseudo Kähler. Weyl Manifolds, IJGMMP, Vol. 10, No. $5 ; 2013 ; 1-8$.
[9] R. Ye, Filling, By Holomorphic Curves In Symplectic 4 Man folds, Trans actions of The American Mathematical Society, Vol. 350, No. 1, 1998, pp. 213-250

[10] New lander, A.; Nirenberg, L. (1957), "Complex analytic coordinates in almost complex manifolds", Annals of Mathematics. Second Series, 65 (3): 391-404, doi: 10. 2307/ 1970051, ISSN 0003-486X, JSTOR 1970051, MR 0088770.

[11] Zeki KASAP, Hamilton Equations on a Contact 5 Manifolds, Elixir Adv. Math. 92 (2016) 38743-38748.

[12] P. J. Higgins, K. Mackenzie: Algebraic constructions in the category of Lie algebraist, J. Algebra, 129 (1990), 194-230.

[13] Z. Kasap, Weyl-Mechanical Systems on Tangent Manifolds of Constant W Sectional Curvature, Int. J. Geom. Methods Mod. Phys. Vol. 10, No. 10; 2013.

[14] S. T. Lisi, Applications of Symplectic Geometry to Hamiltonian Mechanics, Department of Mathematics New York University, 2006. 